\title{
SINGULAR INTEGRAL OPERATORS ON THE UNIT CIRCLE ${ }^{1}$
}

\author{
BY JOEL DAVID PINCUS
}

Communicated by N. Levinson, August 9, 1966

Theorem 1. Let $U$ be unitary and of simple spectral multiplicity and let $V$ be a bounded symmetric operator such that $U V-V U=e\left(\cdot, U^{*} e\right)$ where $e$ is cyclic for $U$. Then $V$ is unitarily equivalent to the operator $L$ defined by

$$
L x(\tau)=D(\tau) x(\tau)+\frac{1}{\pi i} \oint_{\sigma(v)} \frac{k(\tau) k^{*}(t)}{t-\tau} x(t) d t
$$

where $D(\tau)$ is an essentially bounded real-valued function defined on $\sigma(U)$, the spectrum of $U$, and $k(\tau)$ is an essentially bounded measurable complex-valued function.

We confine ourselves without essential restriction to the case that $k(t) \neq 0$ almost everywhere on $\sigma(U)$.

Let

$$
A(l, z)=\exp \frac{1}{2 \pi} \int_{-\pi}^{\pi} \int_{-\infty}^{\infty} \frac{e^{i \theta}+z}{e^{i \theta}-z} g\left(\nu, e^{i \theta}\right) \frac{d \nu}{\nu-l} d \theta
$$

where

$$
g\left(\nu, e^{i \theta}\right)=\frac{1}{\pi} \arg \frac{D\left(e^{i \theta}\right)-\nu-i o-\left|k\left(e^{i \theta}\right)\right|^{2}}{D\left(e^{i \theta}\right)-\nu-i o+\left|k\left(e^{i \theta}\right)\right|^{2}} .
$$

LemMa 1.

$$
[A(\bar{l}, \bar{z})]^{-1}=A^{*}\left(l, \frac{1}{z}\right)
$$

LEMMA 2. Let

$$
\phi(\nu, z)=i \exp \int_{-\pi}^{\pi} g\left(\nu, e^{i \theta}\right) \frac{e^{i \theta}+z}{e^{i \theta}-z} d \theta
$$

for $|z|<1$. Then there exists a one-parameter family of positive singular measures, $d \sigma_{\nu}(\cdot)$, of finite total mass for almost all $\nu$, and a real-valued function $\beta(\nu)$ such that

1 This work performed under the auspices of the U.S. Atomic Energy Commission.

2 The complex conjugate of a function $T$ is denoted by $T^{*}$. 


$$
\phi(\nu, z)=i \beta(\nu)+\int_{-\pi}^{\pi} \frac{e^{i \theta}+z}{e^{i \theta}-z} d \sigma_{\nu}(\theta) .
$$

Let $\left\{P_{\nu}^{(j)}(\theta)\right\}_{j=1}^{m(\nu)}$ denote a complete orthonormal set in $L_{2}(\sigma(U)$, $\left.d \sigma_{\nu}(\cdot)\right)$ where $m(\nu) \equiv$ dimension of $L_{2}\left(\sigma(U), d \sigma_{\nu}(\cdot)\right)$. Set

$$
F_{j}(\nu, z)=\left(A(\nu+i o, z) \int_{-\pi}^{\pi} \frac{P_{\nu}^{(j)}(\theta)}{1-z e^{-i \theta}} d \theta .\right.
$$

THEOREM 2 (Evaluation of the spectral multiplicity of $L$ ). Let $M(\nu)$ $=\left\{e^{i \theta}: g\left(\nu, e^{i \theta}\right)=1\right\}$. If $M(\nu)$ is the union of $n$ disjoint arcs, then $m(\nu)$ $=n$; otherwise, $m(\nu)$ is infinite.

THEOREM 3. Let

$$
\begin{aligned}
P_{\nu}(x, y)=\frac{1}{2 \pi i} \lim _{\eta \downarrow 0}\left(\frac{k\left(e^{i \theta}\right)}{1-x e^{-i \theta}},(L-\nu-i \eta)^{-1}\right. & \\
& \left.-(L-\nu+i \eta)^{-1} \frac{k\left(e^{i \theta}\right)}{1-y e^{-i \theta}}\right)
\end{aligned}
$$

for $|x|<1,|y|<1$, where $(f, g)=\int_{-x}^{\pi} f\left(e^{i \theta}\right) g^{*}\left(e^{i \theta}\right) d \theta$.

Then,

$$
P_{r}(x, y)=\sum_{j=1}^{m(p)} F_{j}(\nu, x) F_{j}^{*}(\nu, y),
$$

and

$$
\int_{\sigma(L)} P_{\nu}(x, y) d \nu=\left(\frac{k\left(e^{i \theta}\right)}{1-x e^{-i \theta}}, \frac{k\left(e^{i \theta}\right)}{1-y e^{-i \theta}}\right) .
$$

The proof of this theorem follows from a residue calculation and the algebraic relations

$$
\begin{aligned}
i\left(\frac{A(\xi+i o, x)}{A(\xi+i o, y)}-\frac{A(\xi-i o, x)}{A(\xi-i o, y)}\right) & \equiv q(\xi, x, y), \\
\frac{1}{2} \frac{1}{1-x \bar{\omega}} q\left(\xi, x \frac{1}{\bar{\omega}}\right) & =\sum_{1}^{m(\xi)} F_{j}(\xi, x) F_{j}^{*}(\xi, \omega)
\end{aligned}
$$

using Lemmas 1 and 2.

This last theorem can be written in the form of an eigenfunction expansion. Thus, set

$$
x_{j}(\xi, \theta)=\frac{1}{k\left(e^{i \theta}\right)} \lim _{\eta \downarrow 0}\left[F_{j}\left(\xi,(1+\eta) e^{i \theta}\right)-F_{j}\left(\xi,(1-\eta) e^{i \theta}\right)\right]
$$


and

$$
S_{j} f(\xi) \equiv \int_{-\pi}^{\pi} f(\theta) x_{j}^{*}(\xi, \theta) d \theta
$$

whenever $f(\theta)$ belongs to the domain of the absolutely continuous part of $L$, the integral existing in the mean square sense, with

$$
S f(\xi) \equiv\left\{S_{1} f(\xi), \cdots, S_{m(\xi)} f(\xi)\right\} .
$$

THEOREM 4. Let

$$
\left\{g_{1}(\xi), \cdots, g_{m(\xi)}(\xi)\right\}=g(\xi)
$$

be a vector in the direct integral Hilbert space $\mathrm{C}^{*}$ formed with respect to Lebesgue measure and the multiplicity function $m(\xi)$. Let

$$
T g(\theta)=\int_{\sigma(V)} \sum_{1}^{m(\nu)} g_{j}(\nu) x_{j}(\nu, \theta) d \nu .
$$

Then $S T=1$ and $T S=1$, and

$$
\int_{\sigma(V)} \sum_{1}^{m(\nu)}\left|g_{j}(\nu)\right|^{2} d \nu=\int_{\sigma(U)}\left|f\left(e^{i \theta}\right)\right|^{2} d \theta .
$$

Furthermore $S L f(\xi)=\xi S f(\xi)$.

The last two theorems imply that $L$, and hence $V$, has an absolutely continuous spectral measure if the spectrum of $U$ is not the entire circle. If the spectrum of $U$ should be the whole unit circle, then the absolutely continuous part of $V$ is diagonalized exactly according to the results presented above. However, in this case when $D\left(e^{i \theta}\right) \pm\left|k\left(e^{i \theta}\right)\right|^{2}=\xi^{ \pm}$are constant, infinitely degenerate eigenmanifolds corresponding to the eigenvalues $\xi^{ \pm}$can appear. Let us see why this is so. If $y(\xi, \tau)$ is an eigenvector of $L$, so that $L y(\xi, \tau)=\xi y(\xi, \tau)$ we have

$$
[D(\tau)-\xi] y(\xi, f)+\frac{1}{\pi i} \oint_{-\pi}^{\phi \pi} \frac{k(\tau) k^{*}(t)}{t-\tau} y(\xi, t) d t .
$$

From this, we may conclude that

$$
\left[D(\tau)-\xi+|k(\tau)|^{2}\right]_{\phi}\left(\xi, \tau^{+}\right)=\left[D(\tau)-\xi-|k(\tau)|^{2}\right]_{\phi}\left(\xi, \tau^{-}\right)
$$

where 


$$
\phi(\xi, z)=\frac{1}{2 \pi i} \int_{|\tau|=1} \frac{k^{*}(t)}{t-z} y(\xi, t) d t .
$$

Thus, if $D(\tau)-\xi-|k(\tau)|^{2}=0$, for example, then $\phi\left(\xi, \tau^{+}\right)=0$. But, by the Plemelj formula this means that

$$
\begin{aligned}
\phi\left(\xi, \tau^{+}\right) & =\frac{1}{2} k^{*}(\tau) y(\xi, \tau)+\frac{1}{2 \pi i} \oint_{|t|=1} \frac{k^{*}(t) y(\xi, t)}{t-\tau} d l \\
& =\frac{1}{2}\left(I+B_{U}\right) k^{*} y,
\end{aligned}
$$

where

$$
H_{U} x(\tau)=\frac{1}{\pi i} \int_{\sigma(U)} \frac{x(t)}{t-\tau} d t .
$$

A relatively simple argument now shows that $H_{U}$ has purely absolutely continuous spectrum if $\sigma(U)$ is not the whole circle-and thus $k^{*} y=0$ or $y=0$ in this case; but $H_{U}$ has an infinitely degenerate eigenmanifold associated with the eigenvalues -1 and 1 if $\sigma(U)$ is the whole circle.

An application to the theory of self-adjoint Toeplitz matrices. Let $k\left(e^{i \theta}\right)$ be positive and integrable on $(-\pi, \pi)$. Then if $P$ is the orthogonal projector from $L_{2}(-\pi, \pi)$ to the Hardy space $\mathfrak{H C}^{2}$, we can represent the Toeplitz operator in the form $T f=P k f, f \in \mathcal{F}^{2}[1]$.

If $f \in \mathcal{H}^{2}$, then $k f \in L_{2}$ and, in the sense of mean convergence

$$
k\left(e^{i \theta}\right) f\left(e^{i \theta}\right)=\sum_{n=-\infty}^{\infty} a_{n} e^{i n \theta}
$$

where

$$
\sum_{n=-\infty}^{\infty}\left|a_{n}\right|^{2}<\infty
$$

but

$$
\sum_{n=0}^{\infty} a_{n} z^{n}=\frac{1}{2 \pi i} \int_{|t|-1} \frac{k(t) f(t)}{t-z} d t, \quad|z|<1 .
$$

Thus

$$
\lim _{r} \sum_{n=0}^{\infty} a_{n} r^{n} e^{i n \theta}=P k\left(e^{i \theta}\right) f\left(e^{i \theta}\right) .
$$

But the Plemelj formula can be used to evaluate this limit, and we obtain 


$$
T f=\frac{1}{2} k\left(e^{i \theta}\right) f\left(e^{i \theta}\right)+\frac{1}{2 \pi i} \oint_{|\tau|=1} \frac{k(\tau) f(\tau)}{\tau-\exp (i \theta)} d \tau .
$$

Let $T$ as written on the right-hand side above be considered as a self-adjoint operator on $L_{2}(k)=L_{2}\left(-\pi, \pi ; k\left(e^{i \theta}\right) d \theta\right)$. Let us denote the closure in this space of finite linear combinations of the form $\sum_{n=0}^{N} b_{n} e^{i n \theta}$ by $\mathcal{T C}_{\mathrm{k}}^{2}$.

Then, if $x \in\left(\mathcal{H C}_{k}^{2}\right)^{\perp}$,

$$
(T x, y)_{L_{2}(k)}=(x, T y)_{L_{2}(k)}=0, \quad \forall y \in L_{2}(k) .
$$

Thus $\left(\mathcal{C}_{k}^{2}\right)^{\perp}$ is the null manifold of $T$.

The reader will see that the spectral analysis of $T$ restricted to $\mathcal{F}_{k}^{2}$ is carried out immediately by an easy application of the results of the preceding section. For a different approach see [2], [3].

FInAL REMark. The proof of our main result is carried out by means of a reduction to a new general theory of singular RiemannHilbert boundary value problems:

$$
\phi^{+}(\xi, \lambda)=G(\xi, \lambda) \phi^{-}(\xi, \lambda)
$$

where

$$
G(\xi, \lambda)=1+\int_{-\infty}^{\infty} \frac{d M_{\lambda}(\nu)}{\nu-\xi-i o}
$$

and $d M_{\lambda}(\cdot)$ is a one-parameter purely singular positive measure [4]. This reduction also makes it possible to give a much more transparent deduction of the author's previous results about singular integral equations on the line [5]. Furthermore, it leads to a spectral theory for self adjoint coupled systems of singular integral equations [6].

\section{REFERENCES}

1. P. R. Halmos, A glimpse into Hilbert space, Vol. 1, Lectures on Modern Mathematics, (T. Saaty, ed.), Wiley, New York, 1963.

2. R. S. Ismagilov, The spectrum of Toeplitz matrices, Dokl. Akad. Nauk SSSR 150 (1963), 769-772 = Soviet Math. Dokl. 4 (1963), 462-465.

3. Marvin Rosenblum, A concrete spectral theory for self-adjoint Toeplitz operators, Amer. J. Math. 87 (1965), 709-718.

4. J. D. Pincus, A singular Riemann-Hilbert problem, 1965 Summer Institute on Spectral Theory and Statistical Mechanics, Brookhaven National Laboratory, Upton, N. Y.

5. - Commutators, generalized eigenfunction expansions and singular integral operators, Trans. Amer. Math. Soc. 121 (1966), 358-377.

6. - Commutators and systems of singular integral equations, (to appear).

BROOKHAVEN NATIONAL LABORATORY 